\title{
An eigenvector spatial filtering contribution to short range regional population forecasting
}

\author{
Daniel A. Griffith* • Yongwan Chun
}

University of Texas at Dallas, USA

Received: 8 October 2014

Revised: 15 December 2014

Accepted: 28 December 2014

\begin{abstract}
Statistical space-time forecasting requires sufficiently large time series data to ensure high quality predictions. The dominance of temporal dependence in empirical space-time data emphasizes the importance of a lengthy time sequence. However, regional space-time data often have a relative small temporal sample size, increasing chances that regional forecasts might result in unreliable predictions. This paper proposes a method to improve regional forecasts by incorporating spatial autocorrelation in a generalized linear mixed model framework coupled with eigenvector spatial filtering. This methodology is illustrated with an application to regional population forecasts for South Korea.
\end{abstract}

Keywords: eigenvector spatial filter, population, South Korea, space-time forecasting, spatial autocorrelation

JEL Classification Codes: C21, P23, R23

\section{Introduction}

Consider a geographic landscape comprising $n$ areal units (e.g., counties) and $T$ points in time. This is a panel data structure (Baltagi, 2013). Space-time forecasting of population counts is an important problem that can be solved statistically with a sufficiently large dataset, or with population projection matrices coupled with a sequence of $T-1 n$-by- $n$ inter-areal unit migration flows data. Because space-time statistical forecasts tend to be dominated by time dependence

\footnotetext{
* Corresponding author. E-mail: dagriffith@utdallas.edu.

Citation: Griffith, D.A. and Chun. Y. (2014) An eigenvector spatial filtering contribution to short range regional population forecasting, Economics and Business Letters, 3(4), 208-217.
} 
(Griffith, 2013), $T$ needs to be relatively large. Meanwhile, as $n$ increases, geographic heterogeneity compromises the simplicity of a space-time statistical forecast. In contrast, collecting areal unit migration data is an onerous and costly task that dissuades the construction of population projection matrix forecasts.

When $T$ is relatively small (e.g., $<<50$ ), and points in time are relatively close together (e.g., 1 year apart), estimated time series statistical forecasting models tend to be unreliable, and resources rarely are spent to repeatedly collect migration data over such a short time horizon. In this context, recognizing that spatial autocorrelation is a manifestation of the inertia-i.e., the resistance to change-in a space-time geographic landscape arising from temporal dependence, spatially structured and spatially unstructured random effects can be estimated separately and then used to make forecasts. The purpose of this paper is to describe this advance in regional forecasting. This furnishes a supplemental approach to panel data analysis for large $n$, small $T$, and unobserved heterogeneity.

\section{Methods}

The set of methods employed for data analysis purposes here includes principal components analysis (PCA), Box-Jenkins time series modelling techniques, eigenvector spatial filtering, and generalized linear mixed model techniques. To adjust for variation in the size of areal units, population counts are converted to densities.

A sequence of annual population density maps for a given geographic landscape tends to display extremely large positive Pearson product moment correlation coefficients. PCA furnishes one multivariate statistical method for confirming this feature of a space-time dataset, and quantifying its magnitude. In this context of highly correlated maps, when the time series is relatively short (e.g., $T=12$ ), an estimated first-order autoregressive parameter, denoted by $\mathrm{AR}(1)$, tends to be near the upper limit of its feasible parameter space (i.e., close to 1). Meanwhile, as $n$ increases, the variability of the $n \operatorname{AR}(1)$ values tends to increase, and more and more areal units tend not to be characterized by the average of these AR(1) values.

Eigenvector spatial filtering (see Chun and Griffith, 2013) employs selected eigenvectors extracted from an $n$-by- $n$ spatial weights matrix $\mathbf{C}$, which articulates the correlation structure for geographic locations across space, as synthetic proxy variables that can be used to control for residual spatial autocorrelation by filtering it out of the model residuals and transferring it to the mean response term. These control variables identify and isolate the stochastic spatial dependencies among a set of georeferenced observations, resulting in independence being mimicked, and thus allowing model estimation to proceed as though the observations are independent. The most popular implementation of eigenvector spatial filtering uses an eigenfunction decomposition of the matrix version of the Moran Coefficient (MC) numerator

$$
\left(\mathbf{I}-\mathbf{1 1}{ }^{\prime} / \mathrm{n}\right) \mathbf{C}\left(\mathbf{I}-\mathbf{1 1}{ }^{\prime} / \mathrm{n}\right)
$$

where $\mathbf{1}$ is an $n$-by-1 vector of ones, and ' denotes the matrix transpose operator. This decomposition generates $n$ eigenvectors, say $\mathbf{E}=\left(\mathbf{E}_{1}, \mathbf{E}_{2}, \mathbf{E}_{3}, \ldots, \mathbf{E}_{\mathrm{n}}\right)$, and their associated $n$ eigenvalues, say $\lambda=\left(\lambda_{1}, \lambda_{2}, \lambda_{3}, \ldots, \lambda_{n}\right)$, where the subscript represents a descending ordering by eigenvalue value. These eigenvalues enable calculation of the MC for their corresponding $n$-by- 1 eigenvectors: $\mathrm{MC}_{\mathrm{j}}=\frac{\mathrm{n}}{\mathbf{1}^{\mathrm{T}} \mathbf{C} \mathbf{1}} \cdot \lambda_{\mathrm{j}}$, for $\mathbf{E}_{\mathrm{j}}, \mathrm{j}=1,2, \ldots, \mathrm{n}$. 
A generalized linear mixed model (GLMM) is a statistical model built upon both normal and non-normal probability models, with a mean response whose relationship with a linear combination of covariates and a random effects term is defined by a link function, say $\mathrm{g}$ (McCulloch, Searle, and Neuhaus, 2008). For simplicity, these random effects frequently are assumed to conform to a zero mean normal distribution. They often are specified with both spatially structured and unstructured components for georeferenced data. Let $Y_{i}$ be a response variable, $\mathbf{X}_{\mathrm{i}}$ be a 1-by- $(p+1)$ vector of $p$ covariates and a 1 (for the intercept term), and $\mathbf{Z}_{\mathrm{i}}$ be a design matrix (e.g., defining repeated measures through time) for observation $i$. Then

$$
\mathrm{E}\left(\mathrm{Y}_{\mathrm{i}}\right)=\mu_{\mathrm{i}}=\mathrm{g}^{-1}\left(\mathbf{X}_{\mathrm{i}} \boldsymbol{\beta}_{\mathrm{X}}+\mathbf{Z}_{\mathrm{i}} \gamma\right)=\mathrm{g}^{-1}\left(\mathbf{X}_{\mathrm{i}} \boldsymbol{\beta}_{\mathrm{X}}+\mathbf{E}_{\mathrm{k}, \mathrm{i}} \boldsymbol{\beta}_{\mathrm{E}}+\xi_{\mathrm{i}}+\mathbf{W}_{\mathrm{i}}\right)
$$

where $E$ denotes the calculus of expectation operator, $\boldsymbol{\beta}_{\mathrm{X}}$ is a $(p+1)$-by-1 vector of covariate regression coefficients (including the intercept term), $\boldsymbol{\beta}_{\mathrm{E}}$ is a $k$-by-1 vector of selected eigenvector regression coefficients, with $\mathbf{E}_{k, i} \boldsymbol{\beta}_{\mathrm{X}}$ being a spatially structured random effects term, $\xi$ is a spatially unstructured random effects term, and $\mathbf{W}_{\mathrm{i}}$ is the sum of $\mathrm{q}>0$ offset variables (which, by definition, have coefficients of 1 ).

\section{Data}

The foundational space-time dataset is for 12 years of resident population counts (1997-2008) across South Korea's $230 \mathrm{Si}-\mathrm{Gun}-\mathrm{Gu}$ (which are similar to counties). These population counts are available from a South Korean government database containing the residential locations of people in South Korea. This resident registration is compulsory in the country, and address changes by residents must be reported to the government. ${ }^{1}$ Jeju Iisland is partitioned into two SiGun-Gu that are not linked to the mainland in the geographic weights matrix. Figure 1a portrays aggregate annual population growth for South Korea as a whole across the 1992-2013 time horizon, together with the Korean quinquennial census population counts since 1925. The two overlapping trajectories are nearly parallel, and roughly linearly related to time (Figure 1a). Because the resident population includes people who temporarily live in other counties due to, for example, their jobs or being students, resident population is slightly larger than its corresponding census population counts.

\footnotetext{
${ }^{1}$ Three different national population statistics are available in South Korea: (1) resident population from the Ministry of Security and Public Administration, (2) census population from Statistics Korea, the Korean national statistical office, and (3) population projections from Statistics Korea. These data can be accessed at http://kosis.kr/statisticsList/statisticsList_01List.jsp.
} 
Figure 1. Statistical characteristics of the space-time series
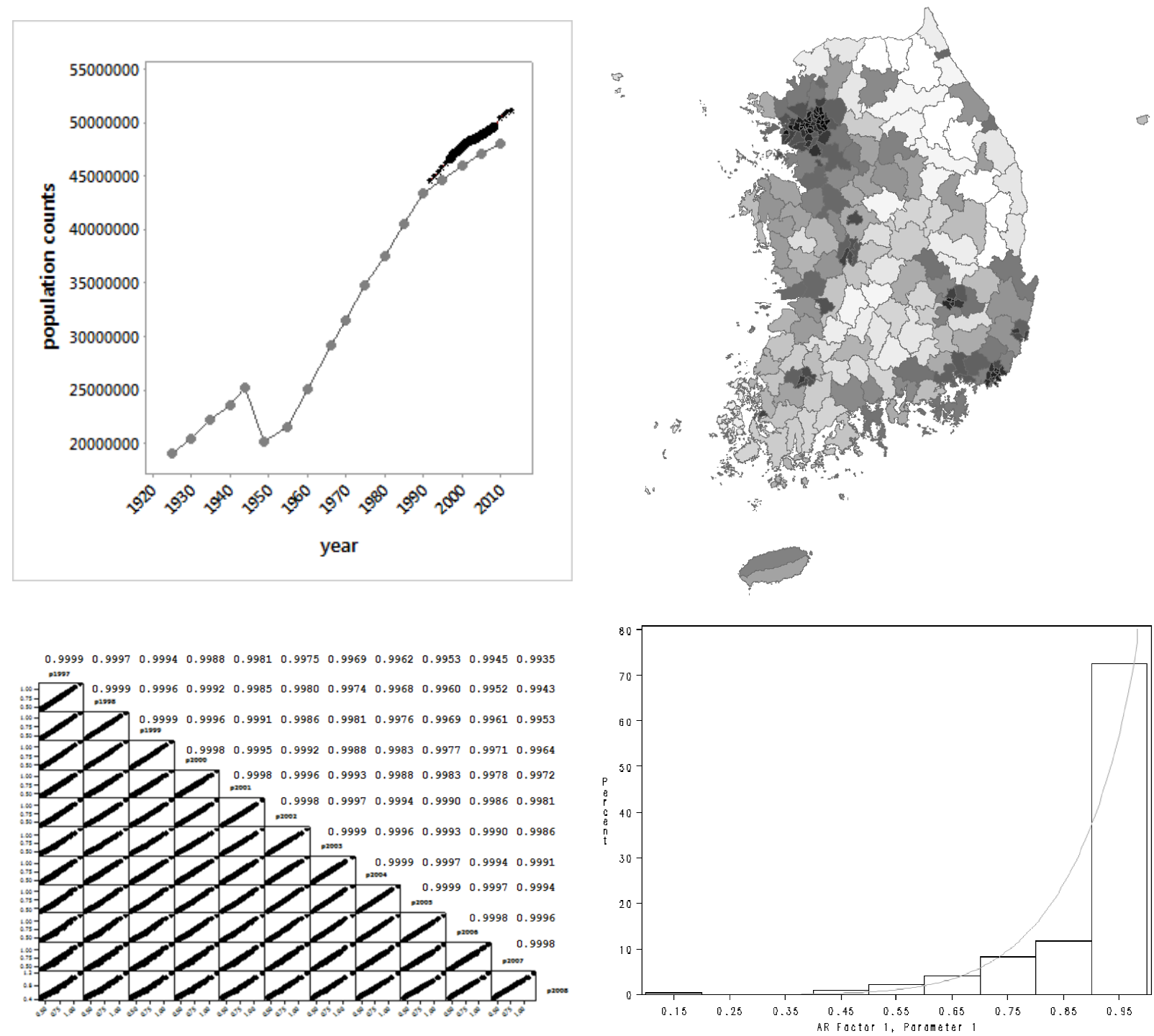

Top left (a): the aggregate South Korea population time series: gray denotes quinquennial census counts, black asterisks denotes resident population counts, and solid black circles denote the foundation years used to estimate the space-time forecasting model.

Top right (b): the geographic distribution of the first principal component scores $(\mathrm{MC}=0.76297)$; score values are directly related to grayscale darkness.

Bottom left (c): scatter plot and correlation matrix for the 12 maps.

Bottom right (d): the frequency distribution of the AR(1) coefficients, with a superimposed beta distribution curve. 


\section{Results}

The population densities (population/area) were subjected to a Box-Cox power transformationnamely, $1 /(\text { density-1.76) })^{0.11}$ — so that they better conform to a bell-shaped curve, a procedure that moderates impacts of outliers ${ }^{2}$. The individual year translation and exponent parameters display a time trend (Table 1). A PCA of the 12 transformed population density maps produces a single component accounting for $99.85 \%$ of the variance in these maps. In other words, the 12 maps are highly multicollinear, and are nearly identical in their map patterns (Figure 1c). Figure 1b portrays the composite map pattern for these transformed population densities.

Table 1. Selected estimation results for the individual population maps

\begin{tabular}{|c|c|c|c|c|c|c|}
\hline \multirow[b]{2}{*}{ Year } & \multicolumn{2}{|c|}{ Box-Cox power transformation } & \multicolumn{4}{|c|}{ Regression including the random effects term } \\
\hline & Translation parameter & exponent & $\begin{array}{l}\text { Dispersion } \\
\text { parameter }\end{array}$ & a & $\mathrm{b}$ & Pseudo-R ${ }^{2}$ \\
\hline 1997 & -1.70 & -0.15 & 0.0134 & -5.7201 & 1.0247 & 0.9946 \\
\hline 1998 & -1.73 & -0.14 & 0.0099 & -4.2765 & 1.0147 & 0.9967 \\
\hline 1999 & -1.74 & -0.14 & 0.0063 & -2.8525 & 1.0103 & 0.9981 \\
\hline 2000 & -1.75 & -0.13 & 0.0035 & -2.2257 & 1.0127 & 0.9991 \\
\hline 2001 & -1.74 & -0.12 & 0.0016 & -0.7317 & 1.0071 & 0.9993 \\
\hline 2002 & -1.73 & -0.11 & 0.0005 & 0.3674 & 1.0018 & 0.9995 \\
\hline 2003 & -1.73 & -0.11 & 0.0007 & 1.2073 & 0.9970 & 0.9996 \\
\hline 2004 & -1.79 & -0.10 & 0.0015 & 1.6840 & 0.9939 & 0.9991 \\
\hline 2005 & -1.83 & -0.09 & 0.0030 & 2.2085 & 0.9894 & 0.9987 \\
\hline 2006 & -1.83 & -0.08 & 0.0052 & 2.6496 & 0.9858 & 0.9981 \\
\hline 2007 & -1.81 & -0.08 & 0.0077 & 3.2487 & 0.9838 & 0.9976 \\
\hline 2008 & -1.79 & -0.08 & 0.0122 & 4.4411 & 0.9788 & 0.9967 \\
\hline overall & -1.76 & -0.11 & 0.0060 & 0.0000 & 1.0000 & 0.9979 \\
\hline
\end{tabular}

Next, AR(1) models were estimated for each of the $n=230(T=12)$ time series of these transformed values (Figure 1d). Over 50\% of the serial correlation parameter estimates exceed 0.95 in value. But some of the values are as small as roughly 0.16 . Moreover, the distribution of the values conforms well to a Beta random variable with parameters 8.1 and 0.9 . These properties coupled with only 12 points in time are antithetical to establishing sound time series forecasting.

Consequently, Eq. 2 was estimated, with the response variable population being a count, and the covariates being a log-area offset variable (i.e., its coefficient was set to 1) - this specification is equivalent to an untransformed population density described by a non-normal probability model - and the intercept term. The link function $\mathrm{g}$ was defined in terms of a negative binomial (i.e., Poisson with a gamma distributed mean) probability model. This specification accounts for nearly all of the variability in population density across South Korea (Table 1). The random effects term has a mean of -0.0060 , but deviates from normality according to its Shapiro-Wilk diagnostic statistic. Its spatially structured component (Figure 2a) is an eigenvector spatial filter (ESF) constructed with 25 vectors that account for $80 \%$ of its variability. Its spatially unstructured component (Figure $2 b$ ) still has a mean of -0.0060 , but conforms closely to a normal

\footnotetext{
${ }^{2}$ The Shapiro-Wilk normality diagnostic statistics increases from 0.67 to 0.95 , which is dramatically closer to 1 . Its standard deviation across the years decreases from 0.006 to 0.001 . Roughly $8.7 \%$ of the original data can be classified as large outliers. The Box-Cox transformation alters the measurement scale in such a way that outliers shrink by about $42 \%$, through to small values being inflated by $151 \%$. The outcome is frequency distributions that more closely mimic normal curves.
} 
distribution (its Shapiro-Wilk statistic probability is 0.2465 ); this term represents heterogeneity across the South Korean geographic landscape. These individual spatially structured and unstructured random effects terms are the same for every point in time, which accounts for the strong pairwise correlations among the twelve maps (Figure 1c). Respecifying the random effects term with a space-time filter (Griffith, 2012) fails to change these results, an outcome that is consistent with the preceding PCA and AR(1) findings.

Figure 2. The random effects terms

Left (a): the spatially structured random effects $(\mathrm{MC}=0.98259)$

Right (b): the spatially unstructured fandom effects $(\mathrm{MC}=0.04931)$
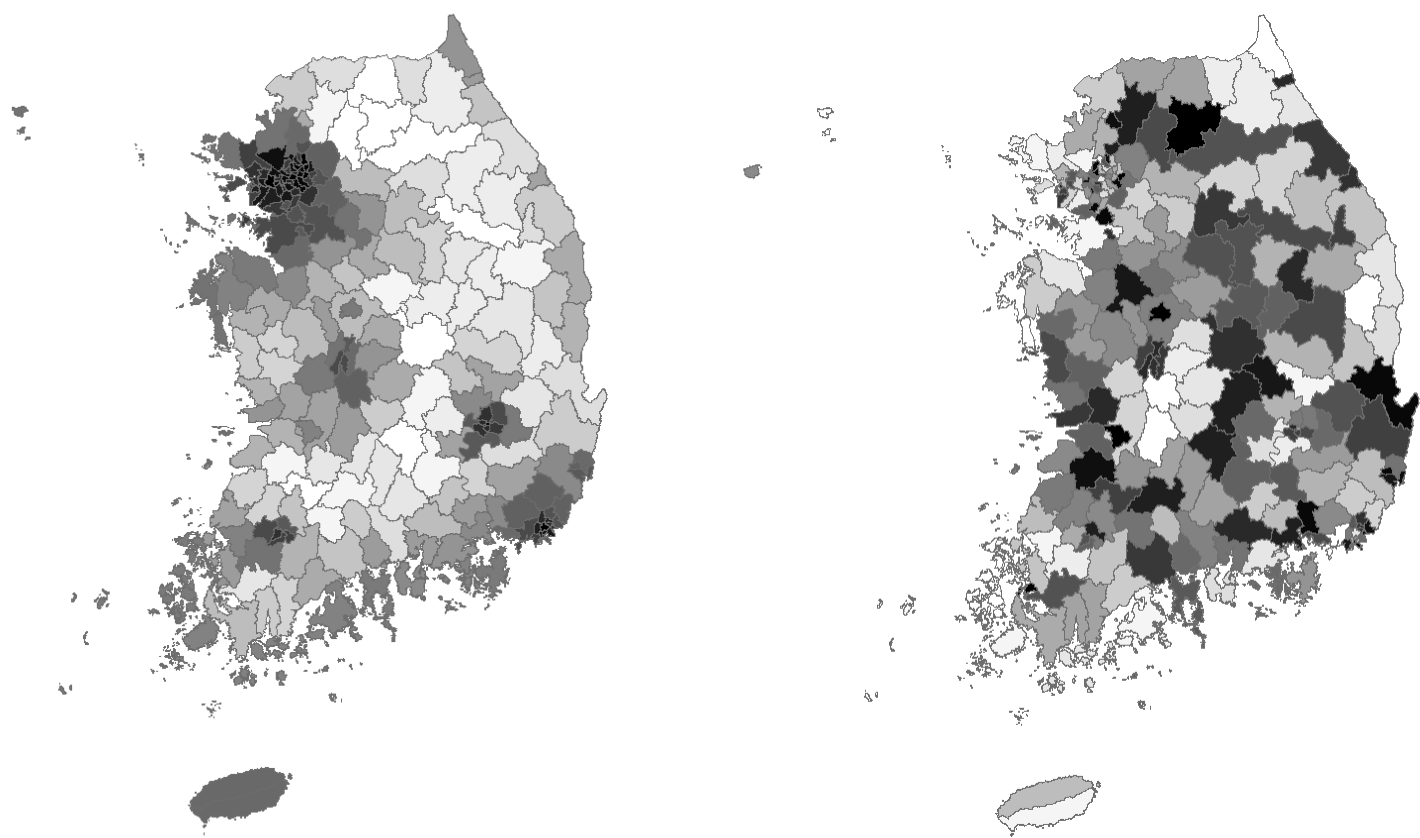

Areal unit values are directly proportional to grayscale darkness.

Griffith and Paelinck (2009) discuss the use of Eq. 2 for space-time forecasting purposes. The bivariate regression coefficients appearing in Table 1 suggest that although the quality of these forecasts decreases through time, which is anticipated, one should expect to get reasonably good forecasts for at least three or four future years (Figure 3a). One reason these forecasts do not extend further into the future is because the random effects term best fits the average point of the series, which tends to be at its midpoint, resulting in a quadratic relationship between time and the $\mathrm{R}^{2}$ values. Nevertheless, although acknowledging a slight time series trend in the bivariate GLMM regression coefficients for the intercept (decreasing from 4.1855 to 4.1633 ) and the ESF aggregate coefficient (increasing from 0.9848 to 1.0136), short range population forecasts can be achieved with the pooled estimated constant term $\mathrm{e}^{4.1725}$ modified by deviations from this mean geographically distributing the population across the country according to areal unit size, the spatial autocorrelation pattern captured by the ESF, and local heterogeneity, all of which are quantities varying across locations but not time. The parameter time trends (Figure 3b) suggest that the constant term becomes less important, whereas the spatial autocorrelation and local 
heterogeneity terms become more important, with the passing of time. Therefore, forecasts can be constructed for time $\mathrm{t}+\mathrm{k}$ by rescaling the estimated model results as follows:

$$
\hat{\mathrm{P}}_{\mathrm{i}, \mathrm{t}+\mathrm{k}}=\operatorname{Area}_{\mathrm{i}} \exp \left(4.1725+\mathrm{ESF}_{\mathrm{i}}+\hat{\xi}_{\mathrm{i}}\right) \frac{\sum_{\mathrm{i}=1}^{230} \mathrm{P}_{\mathrm{i}, \mathrm{t}+\mathrm{k}}}{\sum_{\mathrm{i}=1}^{230} \operatorname{Area}_{\mathrm{i}} \exp \left(4.1725+\mathrm{ESF}_{\mathrm{i}}+\hat{\xi}_{\mathrm{i}}\right)},
$$

where $\mathrm{P}_{\mathrm{i}, \mathrm{t}}$ is the population of areal unit $\mathrm{i}$ are time $\mathrm{t}$, Area $_{\mathrm{i}}$ is the area of areal unit $\mathrm{i}$, and $\mathrm{ESF}_{\mathrm{i}}$ is the ESF value for areal unit i. Eq. 3 requires a total population figure, $\mathrm{P}_{\mathrm{t}+\mathrm{k}}=\sum_{\mathrm{i}=1}^{230} \mathrm{P}_{\mathrm{i}, \mathrm{t}+\mathrm{k}}$, which can be a short range aggregate forecast. Mean squared error (MSE) calculations based upon Eq. 3 again emphasize that the estimated spatially structured and spatially unstructured random effects terms best describe the midpoint of the space-time series (Figure 4a), and hence the equation's restriction to producing only short range forecasts, which becomes a necessary tool when time series are very short. This conclusion does not change by using the year-specific bivariate GLMM coefficients because the random effects terms are estimated with the entire foundational time series. For example, the 2008 year-specific results yield an increase in the MSE of $12.4 \%$. The best case scenario is for 2006, for which the MSE decreases, but by only $1.1 \%$. Spatially structured random effects can be estimated without a time series, although a single-year estimate may contain some biased; but the spatially unstructured random effects term, which here accounts for roughly $20 \%$ of the variability in the total random effects term, cannot.

Figure 3. Time series for model goodness-of-fit statistics and parameter estimates for population densities

Left (a): forecasted $\mathrm{R}^{2}$ values with prediction intervals.

Right (b): individual map bivariate regression coefficients for the random effect terms; black denotes the coefficient for the spatially structured term, and gray denotes the coefficient for the spatially unstructured term.
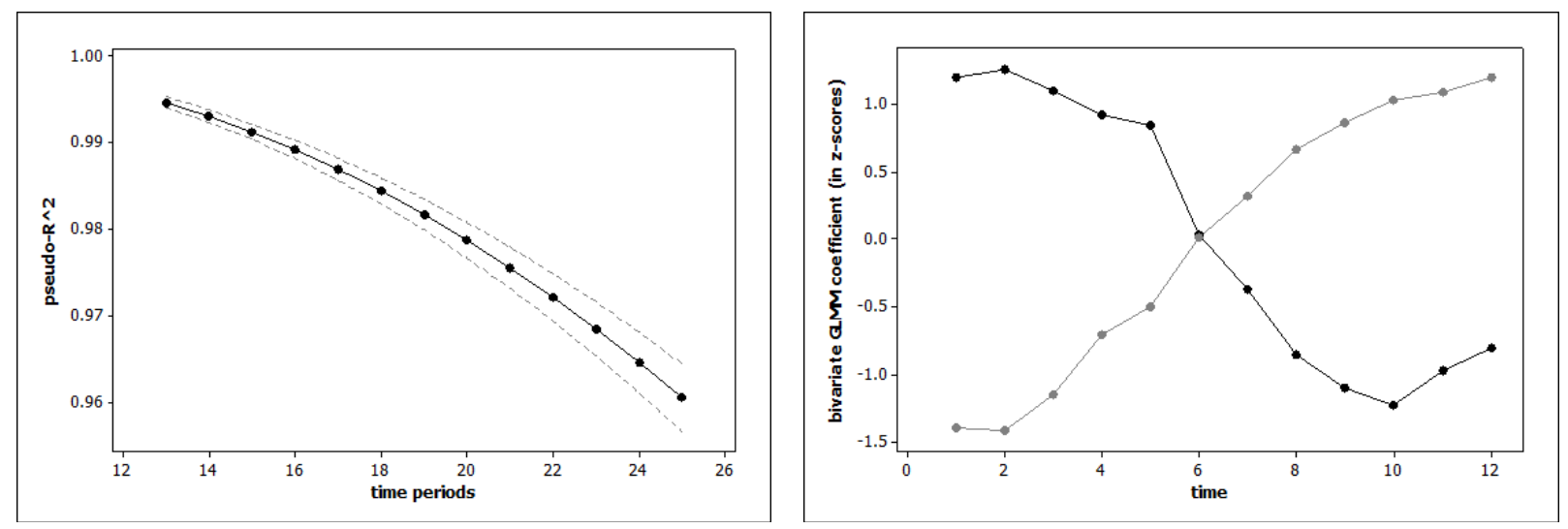
Figure 4. Time series for model goodness-of-fit and population density forecast prediction error

Left (a). The MSE for the population maps based upon the forecast Eq. 3

Right (b). Scatter plot and correlation matrix for the spatial prediction error and five forecast error maps
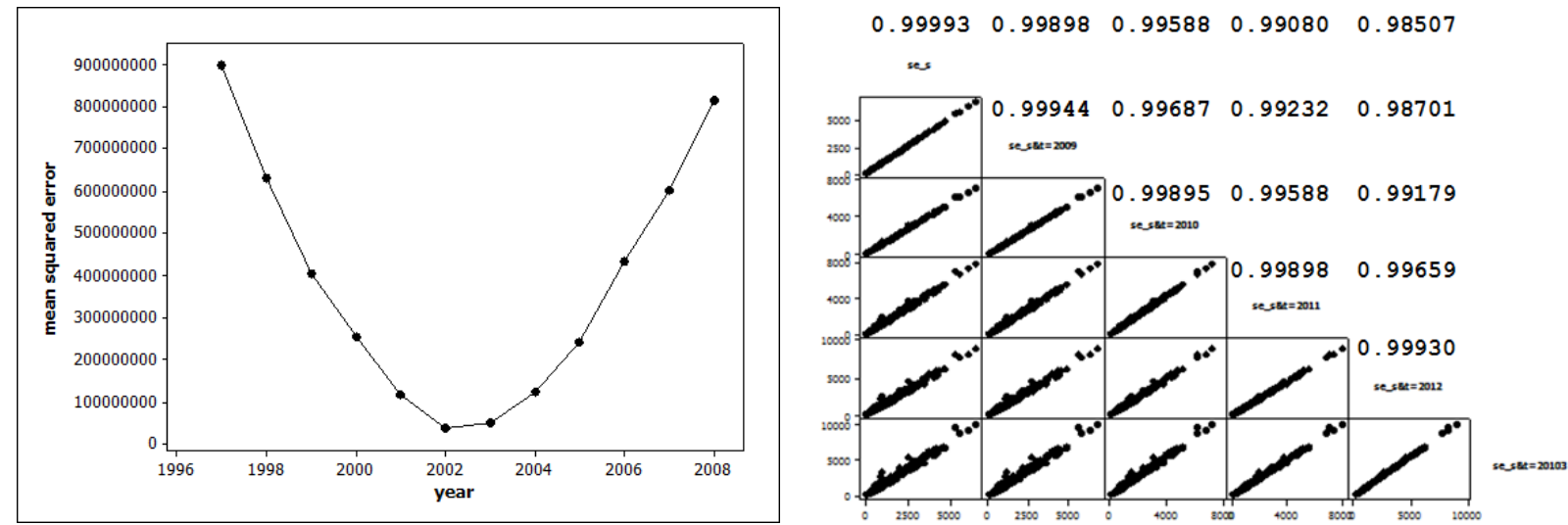

The annual space-time results for 2009-2013 can be analyzed to assess the quality of population count estimates presented by Eq. 3 estimated with data for 1997-2008. A simple BoxJenkins time series forecast based upon the annual total population counts for South Korea during 1992-2008 (Figure 1a) yields

$$
\hat{\mathrm{P}}_{\mathrm{t}}=(1-0.85401) \times 344381.2+(1-0.85401) \mathrm{P}_{\mathrm{t}-1}+0.85401 \mathrm{P}_{\mathrm{t}-2},
$$

where $\mathrm{P}_{\mathrm{t}}$ denotes the population of South Korea at time $\mathrm{t}$, a first differencing was done, the firstorder autoregressive parameter estimate is 0.85401 , and the mean of the differenced series (with only 17 points in time) is 344381.2 . Residual diagnostics suggest that only trace serial correlation remains. Table 2 reports the forecasts for the subsequent five years, which are reasonably good given the shortness of the time series. The pseudo- $\mathrm{R}^{2}$ values are better than expected (Figure $3 \mathrm{a}$ ), and are identical for the observed and forecasts because the forecasts are simply a rescaling of the predicted values corresponding to the observed counts. On average, the MSEs for the forecasts are more than $20 \%$ greater than their corresponding MSEs for the predicted values. This reflects the sizeable prediction error for the forecasted 1997-2008 total population: the variance of the space-time distribution of values has an error term for the forecasts multiplied by an error term for the predicted values; this latter error component is constant over time, whereas this former error component increases across a forecast horizon. Figure 5a presents the 2009 space-time population counts forecast; Figure 5b portrays the geographic distribution of its spatial prediction error. This result can be extended to space-time forecasts by the mathematical statistics theorem (Goodman, 1962) about the variance of the product of two independent random variables (i.e., the time series forecasts here are independent of the spatial forecasts), which equals

$$
\frac{\sigma_{\mathrm{s}_{\mathrm{i}}}^{2} \sigma_{\mathrm{T}}^{2}+\mu_{\mathrm{s}_{\mathrm{i}}}^{2} \sigma_{\mathrm{T}}^{2}+\mu_{\mathrm{T}}^{2} \sigma_{\mathrm{s}_{\mathrm{i}}}^{2}}{\sum_{\mathrm{i}=1}^{230} \operatorname{Area}_{\mathrm{i}} \exp \left(4.1725+\mathrm{ESF}_{\mathrm{i}}+\hat{\xi}_{\mathrm{i}}\right)},
$$

where the denominator $\sum_{\mathrm{i}=1}^{230} \operatorname{Area}_{\mathrm{i}} \exp \left(4.1725+\mathrm{ESF}_{\mathrm{i}}+\hat{\xi}_{\mathrm{i}}\right)=48,212,412$ and can be assumed fixed here because it does not change from forecast to forecast, $\sigma_{\mathrm{T}}^{2}$ denotes the variance of the time 
series forecast (from Table 2), $\mu_{\mathrm{T}}$ denotes the forecasted time series value (i.e., its mean; from Table 2), $\sigma_{\mathrm{s}_{\mathrm{i}}}^{2}$ denotes the spatial variance for areal unit $\mathrm{i}$ (portrayed in Figure $5 \mathrm{~b}$ ), and $\mu_{\mathrm{s}_{\mathrm{i}}}$ denotes the spatial mean for areal unit i (portrayed in Figure 5a). The time series forecasting uncertainty approximately multiplies each of the areal unit prediction standard errors by the following factors:

\begin{tabular}{lccccc}
\hline year & 2009 & 2010 & 2011 & 2012 & 2013 \\
\hline factor & 1.05013 & 1.11160 & 1.21758 & 1.36293 & 1.53854 \\
\hline
\end{tabular}

In other words, the map in Figure 5b essentially retains its geographic pattern, but with rescaled values (Figure 4b).

Table 2. Selected summary statistics for the forecasts

\begin{tabular}{cccccrcr}
\hline \hline \multirow{2}{*}{ Year } & \multicolumn{3}{c}{ Total population forecast } & \multicolumn{2}{c}{ Observed total } & \multicolumn{2}{c}{ Forecast total } \\
& Forecast & Standard error & In 95\% CI & $\mathrm{R}^{2}$ & \multicolumn{1}{c}{ MSE } & $\mathrm{R}^{2}$ & \multicolumn{1}{c}{ MSE } \\
\cline { 2 - 8 } 2009 & 49822455 & 60988 & Yes & 0.9956 & 815827242 & 0.9956 & 1032494759 \\
2010 & 50113636 & 128471 & No & 0.9950 & 1034744617 & 0.9950 & 1310487901 \\
2011 & 50412584 & 203292 & Yes & 0.9938 & 1287201916 & 0.9938 & 1566867185 \\
2012 & 50718165 & 282071 & Yes & 0.9932 & 1550314549 & 0.9932 & 1844516609 \\
2013 & 51029410 & 362684 & Yes & 0.9923 & 1833486681 & 0.9923 & 2150340770 \\
\hline \hline
\end{tabular}

Figure 5. The geographic distribution of 2009 space-time-based population count forecasts and their uncertainty

5a. The space-time population forecast for 2009

5b. The spatial prediction standard error
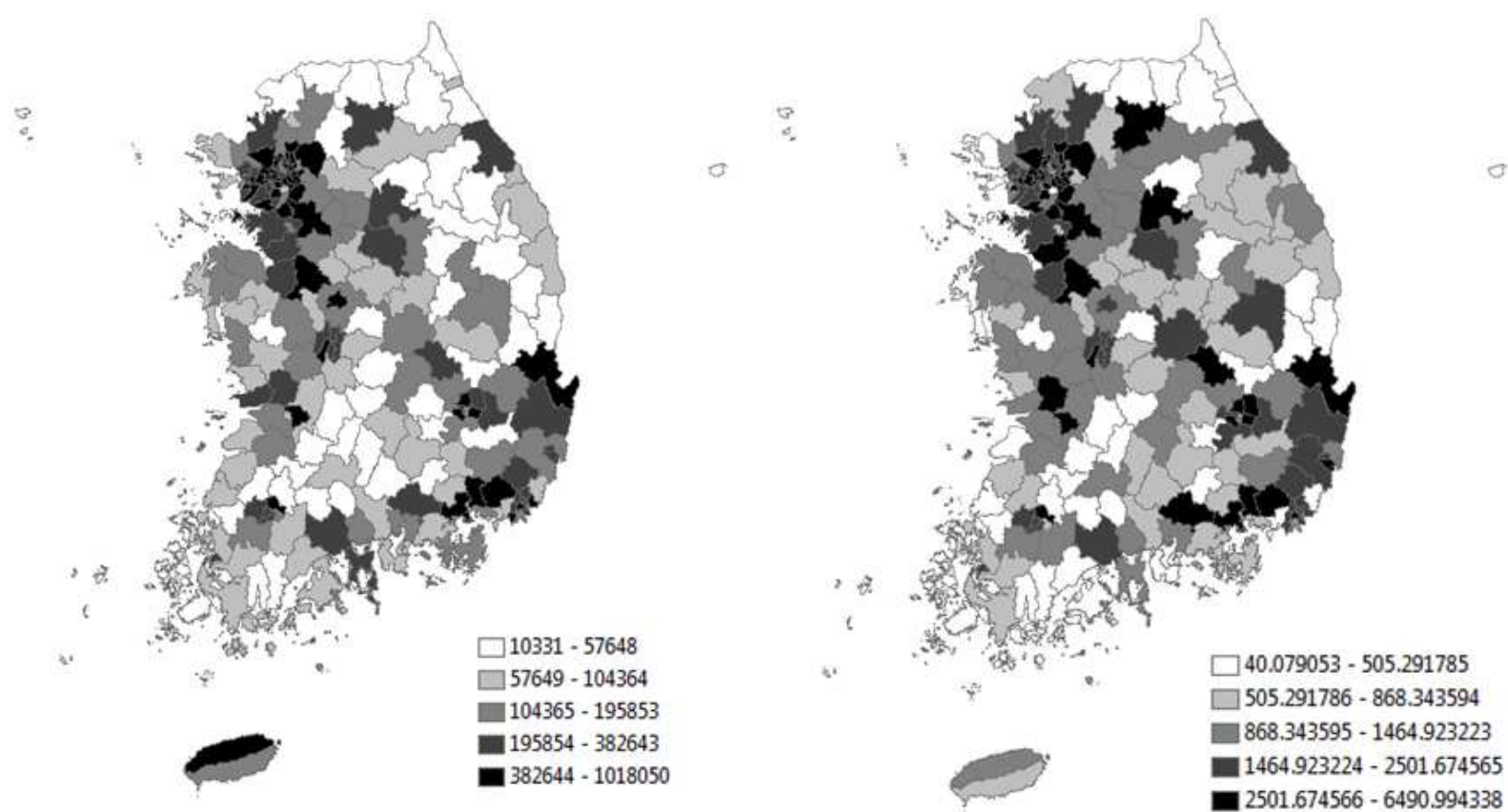


\section{Concluding remarks}

This paper summarizes an advance in short run space-time regional population forecasting when existing time series contain relatively few observations and points in time are close together. This advance exploits spatial autocorrelation as well as random effects in a GLMM framework to geographically distribute aggregate population over areal units of a geographic landscape. The spatial autocorrelation component is approximated with an ESF, which captures the complex map pattern portraying spatial dependence that is latent in population counts, and preserves it in regional forecasts of population. As such, this paper differs from Griffith and Paelinck (2009) in four ways: (1) it treats a substantially larger number of areal units (230 rather than 11); (2) it evaluates forecasts with observed data (rather than output from a spatial econometric model); (3) it derives prediction error maps to accompany population forecast maps; and, (4) it analyzes South Korean population counts (rather than Belgian economic value added figures).

One surprising outcome from the analysis summarized here is that the quality of the regional forecasts is better than expected, given diagnostics such as Figure 3. A second is that the two time components in expression (5) do not dominate the prediction error maps. A third is that although time dependence almost always dominates spatial dependence in practice, results summarized here demonstrate that such temporal dominance can materialize through inertia in spatial dependence as captured by a spatially structured random effects term.

Empirical evaluations of the short run population forecasts indicate that using an ESF to describe spatially structured random effects coupled with a spatially unstructured random effects term furnishes good annual county-level geographic resolution predictions for several years into the future. Similar evaluations need to be conducted for other countries and other geographic resolutions.

Acknowledgements. We thank Monghyeon Lee and Hyeongmo Koo for their assistance in collecting the South Korea population dataset.

\section{References}

Batalgi, B. (2013) Econometric Analysis of Panel Data, $5^{\text {th }}$ ed., Wiley: New York.

Chun, Y. and Griffith, D. (2013) Spatial Statistics \& Geostatistics, SAGE: Thousand Oaks, CA.

Goodman, L. (1962) The variance of the product of K random variables, Journal of the American Statistical Association, 57, 54-60.

Griffith, D. (2012) Space, time, and space-time eigenvector filter specifications that account for autocorrelation, Estadística Española, 54(177), 7-34.

Griffith, D. (2013) Estimating missing data values for georeferenced Poisson counts, Geographical Analysis, 45, 259-284.

Griffith, D. and Paelinck, J. (2009) Specifying a joint space- and time-lag using a bivariate Poisson distribution, Journal of Geographical Systems, 11, 23-36.

McCullogch, C., Searle, S. and Neuhaus, J. (2008) Generalized, Linear, and Mixed Models, $2^{\text {nd }}$ ed., Wiley: New York. 